\title{
Anomalous reaction-transport processes: The dynamics beyond the law of mass action
}

\author{
Daniel Campos, ${ }^{1}$ Sergei Fedotov, ${ }^{1}$ and Vicenç Méndez ${ }^{1,2}$ \\ ${ }^{1}$ School of Mathematics, University of Manchester, Manchester M60 1QD, United Kingdom \\ ${ }^{2}$ Grup de Física Estadística, Departament de Física, Facultat de Ciències, \\ Universitat Autònoma de Barcelona, 08193 Bellaterra, Barcelona, Spain \\ (Received 7 March 2008; revised manuscript received 19 May 2008; published 20 June 2008)
}

\begin{abstract}
In this paper we reconsider the mass action law (MAL) for the anomalous reversible reaction $A \rightleftarrows B$ with diffusion. We provide a mesoscopic description of this reaction when the transitions between two states $A$ and $B$ are governed by anomalous (heavy-tailed) waiting-time distributions. We derive the set of mesoscopic integro-differential equations for the mean densities of reacting and diffusing particles in both states. We show that the effective reaction rate memory kernels in these equations and the uniform asymptotic states depend on transport characteristics such as jumping rates. This is in contradiction with the classical picture of MAL. We find that transport can even induce an extinction of the particles such that the density of particles $A$ or $B$ tends asymptotically to zero. We verify analytical results by Monte Carlo simulations and show that the mesoscopic densities exhibit a transient growth before decay.
\end{abstract}

DOI: 10.1103/PhysRevE.77.061130

PACS number(s): 05.40.Fb, 82.40.-g

\section{INTRODUCTION}

The mass action law (MAL) plays a very important role in a large number of chemical, biological, and physical systems $[1,2]$. It states that the rate of an elementary reaction is proportional to concentrations of reactants. MAL also gives the expression for the equilibrium constant which is a main characteristic of chemical equilibrium. To illustrate this, consider the reversible reaction $A \rightleftarrows B$, with $\beta_{1}$ and $\beta_{2}$ denoting the forward and backward rate constants. According to MAL, the balance equations for the mean concentrations $n_{1}$ and $n_{2}$ of diffusing particles $A$ and $B$ can be written as

$$
\begin{aligned}
& \frac{\partial n_{1}}{\partial t}=D_{1} \nabla^{2} n_{1}-\beta_{1} n_{1}+\beta_{2} n_{2}, \\
& \frac{\partial n_{2}}{\partial t}=D_{2} \nabla^{2} n_{2}+\beta_{1} n_{1}-\beta_{2} n_{2},
\end{aligned}
$$

where $D_{1}$ and $D_{2}$ are the diffusion coefficients of the particles $A$ and $B$, respectively. It follows from Eq. (1) that the uniform equilibrium state $\left(n_{1}^{\infty}, n_{2}^{\infty}\right)$ obeys the equation

$$
\frac{n_{1}^{\infty}}{n_{2}^{\infty}}=\frac{\beta_{2}}{\beta_{1}}=K_{e q},
$$

where $K_{e q}$ represents the equilibrium constant of the reaction process. This constant depends on the thermodynamic properties of the system, but is independent from the transport parameters $D_{1}$ and $D_{2}$. The purpose of this paper is to reconsider these two fundamental equations (1) and (2) for anomalous reaction and transport.

Continuous-time random-walk (CTRW) models have been widely used in recent years to gain insights into the anomalous transport [3]. The extension of CTRW models to reaction-transport phenomena presents modeling challenges, because of the difficulty of taking into account chemical reactions within anomalous transport. Recently several authors have explored the reaction-transport models in which the standard diffusion is replaced by an anomalous (subdiffu- sive) transport [4-11]. It has been shown that the evolution equations for density of particles are drastically different from the standard reaction-diffusion equations. For example, the transport and reaction terms are not separable as it happens in the classical case (1). Instead, one finds that the transport term becomes dependent on the reaction constants $\beta_{1}$ or $\beta_{2}[6,9]$. The master equation for the mean density of one of the reactants may include a crossed transport term [7]. This is a consequence of the non-Markovian nature of subdiffusion.

In previous works, however, reaction was always introduced phenomenologically following the principles of classical reaction kinetics. The idea of this paper is to consider both the reversible reaction $A \rightleftarrows B$ and subdiffusive transport from a probabilistic point view. It is well known that the classical kinetics in Eq. (1) corresponds to Markovian transition of particles from one state to another. Our aim is to take into account anomalous (non-Markovian) transitions of particles from the state $A$ to $B$ and backward and illustrate how the transport process and reactions are coupled. Long tail waiting time distributions describe a variety of phenomena from complex and random materials [3]. Usually chemical reaction with simple kinetic scheme $A \rightleftarrows B$ involves many intermediate reactions. The overall reaction is the result of sequential transitions and each of them is Markovian. They are characterized by the standard exponential waiting time distributions. But the observed overall two-state reaction turns out to be non-Markovian with power-law waiting time distributions. The possibility of long tail kinetics arises because of averaging of exponential waiting time distributions. For example, this phenomenon has been observed in stochastic transitions between closed and open state of ion channel [12].

In what follows we will show that fundamental constant $K_{e q}$ becomes dependent on transport characteristics which is in contradiction to the classical picture of MAL. This is due to the anomalous nature of transitions $A \rightleftarrows B$ for which the waiting time distribution exhibits the power-law decay with the infinite mean waiting time. Let us mention that the situations that are outside the scope of the MAL have already 
been reported for diffusion-limited reactions with long-range interactions in space [13]. The reversible reaction $A \rightleftarrows B$ can be interpreted as a switching between two states $A$ and $B$. This topic has attracted a great interest recently because the switching process can be non-Markovian. Examples include two-state ion channel gating [12], stochastic resonance [14], quantum dots $[15,16]$, etc. For anomalous switching process without transport the mean residence time of the particles in each state is divergent $[15,17]$. As a result, the density of particles in one state tends to zero in the limit $t \rightarrow \infty$ which means the extinction of one of the states. Here we show that the transport process can drastically modify extinctionsurvival dynamics for anomalous transitions. One of the motivations for our study is the experimental data for a malignant brain cancer that exhibits migration-proliferation dichotomy [18]. The motility (transport) of cells and phenotype transitions $A \rightleftarrows B$ (proliferation $\rightleftharpoons$ migration) can be anomalous simultaneously [6]. A possible application of our model is the isomerization reaction for which macromolecules in two interconvertible states migrate with different electrophoretic mobility [19]. Another interesting example is quantum dots (QDs) which are two-state nanocrystals exhibiting anomalous transitions between two states [15,16]. QDs can be used as markers for imaging and other biological applications. Imaging techniques measure the fluorescent light emitted by one of the two states of the QDs. So the experimental results could be affected by the characteristics of the random transition between states. We expect that our model might be useful in interpreting those experiments.

\section{STOCHASTIC MODEL FOR TRANSPORT AND CHEMICAL REACTION}

In this paper we consider the following stochastic model for the transport and reversible reaction $A \rightleftarrows B$. The particles of type $A$ and $B$ randomly move along one-dimensional space and switch between the states $A$ and $B$. This random walk with switching can be described by four sequences of mutually independent random variables. Two sequences describe the waiting times between jumps for particles in the state $A$ and $B$ correspondingly. We assume that these random variables are identically distributed with probability density function (pdf) $\varphi_{1}(t)$ for particles $A$ and pdf $\varphi_{2}(t)$ for particles $B$. Two other sequences describe the waiting times for random transitions: $A \rightarrow B$ and $B \rightarrow A$, respectively. They are identically distributed with pdf's $\psi_{12}(t)$ and $\psi_{21}(t)$, respectively. For example, if we place the particle at the position $x$ at time 0 in state $B$, and if a random time for a jump is less than a random time for reaction $B \rightarrow A$, then the random jump happens. However, if a random time for reaction is less that a random time for a jump, then the transition $B \rightarrow A$ occurs first. In other words, an event (reaction or jump) happens at a time which is the minimum of two random times. So jumps and transitions $A \rightleftarrows B$ are not independent random events as in $[7,11]$.

We express the density of particles $n_{i}(x, t)$ at position $x$ at time $t$ in terms of the initial distribution of particles $n_{i}^{0}(x)$ and density of particles $j_{i}(x, t-\tau)$ that arrive at the same position $x$ at previous time $t-\tau$. The balance equations for the mean densities $n_{1}$ and $n_{2}$ of particles $A$ and $B$ are

$$
\begin{aligned}
& n_{1}(x, t)=n_{1}^{0}(x) \Phi_{1}(t) \Psi_{12}(t)+\int_{0}^{t} j_{1}(x, t-\tau) \Phi_{1}(\tau) \Psi_{12}(\tau) d \tau, \\
& n_{2}(x, t)=n_{2}^{0}(x) \Phi_{2}(t) \Psi_{21}(t)+\int_{0}^{t} j_{2}(x, t-\tau) \Phi_{2}(\tau) \Psi_{21}(\tau) d \tau,
\end{aligned}
$$

where $\Phi_{i}(t)=\int_{t}^{\infty} \varphi_{i}(\tau) d \tau$ and $\Psi_{i j}(t)=\int_{t}^{\infty} \psi_{i j}(\tau) d \tau$ are the corresponding survival probabilities for $\varphi_{i}(t)$ and $\psi_{i j}(t)$. For example, $\Phi_{1}(t)$ is the probability that a particle in the state $A$ does not jump until time $t$, and $\Psi_{12}(t)$ is the probability that a particle in the state $A$ does not switch to $B$ until time $t$. The first term on the right-hand side (RHS) of Eq. (3) represents the contribution from the initial density of particles that have neither jumped nor switched until time $t$. The density $j_{i}(x, t)$ describes how the particles arrive at point $x$ at time $t$ as a result of the transport and switching processes. Equations for the density $j_{1}(x, t)$ of particles $A$ and the density $j_{2}(x, t)$ of particles $B$ can be written as

$$
\begin{aligned}
j_{1}(x, t)= & n_{2}^{0}(x) \Phi_{2}(t) \psi_{21}(t)+\varphi_{1}(t) \Psi_{12}(t) \int_{-\infty}^{\infty} n_{1}^{0}(x-z) \rho_{1}(z) d z \\
& +\int_{0}^{t} j_{2}(x, t-\tau) \Phi_{2}(\tau) \psi_{21}(\tau) d \tau \\
& +\int_{0}^{t} \int_{-\infty}^{\infty} j_{1}(x-z, t-\tau) \varphi_{1}(\tau) \Psi_{12}(\tau) \rho_{1}(z) d z d \tau, \\
j_{2}(x, t)= & n_{1}^{0}(x) \Phi_{1}(t) \psi_{12}(t)+\varphi_{2}(t) \Psi_{21}(t) \int_{-\infty}^{\infty} n_{2}^{0}(x-z) \rho_{2}(z) d z \\
& +\int_{0}^{t} j_{1}(x, t-\tau) \Phi_{1}(\tau) \psi_{12}(\tau) d \tau \\
& +\int_{0}^{t} \int_{-\infty}^{\infty} j_{2}(x-z, t-\tau) \varphi_{2}(\tau) \Psi_{21}(\tau) \rho_{2}(z) d z d \tau . \quad(4)
\end{aligned}
$$

The first equation is the conservation law for particles of type $A$ at time $t$ at position $x$. The first term on the RHS of the equation accounts for the initial distribution $n_{2}^{0}(x)$ of particles in state $B$ that switch to $A$ at time $t$, provided they do not jump up to time $t$. The second term is the contribution from the initial density $n_{1}^{0}(x)$ of particles in state $A$ that jump to $x$ from $x-z$ at time $t$ having not switched until $t$. The random jump length $z$ is distributed by the dispersal kernel $\rho_{1}(z)$. The third term represents the contribution from those particles that switch from state $B$ to state $A$ after a waiting time $\tau$, under the condition that they do not jump during that time. Finally, the fourth term corresponds to the contribution of particles in state $A$ that arrive at $x-z$ at $t-\tau$ that do not switch to $B$ during time $\tau$.

\section{MASTER EQUATIONS}

The set of linear equations (3) and (4) can be solved by using the Laplace-Fourier transforms, $(x, t) \rightarrow(q, s)$. One can obtain two equations, 


$$
\begin{aligned}
s n_{1}(q, s)-n_{1}^{0}(q)= & k_{1}(s)\left[\rho_{1}(q)-1\right] n_{1}(q, s)-a_{1}(s) n_{1}(q, s) \\
& +a_{2}(s) n_{2}(q, s)
\end{aligned}
$$

and

$$
\begin{aligned}
s n_{2}(q, s)-n_{2}^{0}(q)= & k_{2}(s)\left[\rho_{2}(q)-1\right] n_{2}(q, s)+a_{1}(s) n_{1}(q, s) \\
& -a_{2}(s) n_{1}(q, s) .
\end{aligned}
$$

Here we introduce

$$
k_{i}(s) \equiv \frac{\left[\varphi_{i} \Psi_{i j}\right]_{s}}{\left[\Phi_{i} \Psi_{i j}\right]_{s}}, \quad a_{i}(s) \equiv \frac{\left[\Phi_{i} \psi_{i j}\right]_{s}}{\left[\Phi_{i} \Psi_{i j}\right]_{s}},
$$

where $[f]_{s} \equiv f(s)$ denotes Laplace transform. Taking the inverse Laplace and Fourier transforms we obtain the following master equations:

$$
\begin{aligned}
\frac{\partial n_{1}}{\partial t}= & \int_{0}^{t} \int_{-\infty}^{\infty} n_{1}(t-\tau, x-z) k_{1}(\tau)\left[\rho_{1}(z)-\delta(z)\right] d z d \tau \\
& -\int_{0}^{t} a_{1}(\tau) n_{1}(t-\tau, x) d \tau+\int_{0}^{t} a_{2}(\tau) n_{2}(t-\tau, x) d \tau, \\
\frac{\partial n_{2}}{\partial t}= & \int_{0}^{t} \int_{-\infty}^{\infty} n_{2}(t-\tau, x-z) k_{2}(\tau)\left[\rho_{2}(z)-\delta(z)\right] d z d \tau \\
& +\int_{0}^{t} a_{1}(\tau) n_{1}(t-\tau, x) d \tau-\int_{0}^{t} a_{2}(\tau) n_{2}(t-\tau, x) d \tau,
\end{aligned}
$$

where $k_{i}(t)$ and $a_{i}(t)$ are the inverse Laplace transforms of $k_{i}(s), a_{i}(s)$ defined in Eq. (5). The most interesting feature of the system (6) is that effective reaction rate memory kernels $a_{1}(t)$ and $a_{2}(t)$ depend on the transport through the survival probabilities $\Phi_{1}(t)$ and $\Phi_{2}(t)$, while the transport memory kernels $k_{1}(t)$ and $k_{2}(t)$ depend on statistical characteristics of reactions such as $\psi_{i j}$. If the random waiting times for switching and jumping are exponentially distributed, $\varphi_{i}=\lambda_{i} e^{-\lambda_{i} t}$, $\psi_{i j}=\beta_{i} e^{-\beta_{i} t}$, then these dependencies cease to exist. As a result the transport and reaction terms are separable as in the classical case (1). For example, if we use the diffusive approximation for transport $\rho_{i}(q) \sim 1-\sigma_{i}^{2} q^{2}$ (i.e., small jump lengths), then the system (6) can be written as classical reaction-diffusion equations (1), with $D_{i} \equiv \lambda_{i} \sigma_{i}^{2}$. Similarly, for a Markovian switching process with subdiffusive transport, we could recover from Eq. (6) the model for cancer spreading studied in [6]. If the waiting time pdf $\psi_{12}$ has a $\gamma$ distribution as $\psi_{12}=\beta_{1}^{2} t e^{-\beta_{1} t}$ and $\varphi_{1}=\lambda_{1} e^{-\lambda_{1} t}$, then $a_{1}(s)$ $=\beta_{1}^{2}\left(2 \beta_{1}+\lambda_{1}+s\right)^{-1}$. So the reaction-rate memory kernels are

$$
a_{i}(t)=\beta_{i}^{2} e^{-\left(2 \beta_{i}+\lambda_{i}\right) t}, \quad i=1,2 .
$$

This formula shows that the effective reaction rate kernels depend on the rate of jumps $\lambda_{i}$.

\section{A. Asymptotic states as $t \rightarrow \infty$}

Now, let us find the uniform stationary states corresponding to Eq. (6) under the condition $n_{1}^{\infty}+n_{2}^{\infty}=1$. From the limit $q \rightarrow 0$ one finds $\rho_{i}(q)=1$, so we obtain the asymptotic state as $s \rightarrow 0$,

$$
\left(n_{1}^{\infty}, n_{2}^{\infty}\right)=\lim _{s \rightarrow 0}\left(\frac{a_{2}(s)}{a_{1}(s)+a_{2}(s)}, \frac{a_{1}(s)}{a_{1}(s)+a_{2}(s)}\right) .
$$

The main feature of this asymptotic state is that in general it depends on the characteristics of the transport process which is in contradiction with the mass action law (2). This follows from the fact that the survival function $\Phi_{i}$ appears in the definition of $a_{i}$. This happens for any situation except when the switching process is Markovian for which $\lim _{s \rightarrow 0} a_{i}$ $=\beta_{i}$.

Assume now that the reaction process is governed by a power-law decaying distribution of waiting times. We use the standard approximation $\left[\psi_{i j}\right]_{s} \sim 1-\left(\beta_{i}^{-1} s\right)^{\gamma_{i j}}$ as $s \rightarrow 0$ with $\gamma_{i j}<1$. On the contrary, for the transport we consider the Markovian case, $\varphi_{i}(t)=\lambda_{i} e^{-\lambda_{i} t}$. Then, we find that the state (8) turns into

$$
\left(\frac{\lambda_{2}^{1-\gamma_{21}} \beta_{2}^{\gamma_{21}}}{\lambda_{1}^{1-\gamma_{12}} \beta_{1}^{\gamma_{12}}+\lambda_{2}^{1-\gamma_{21}} \beta_{2}^{\gamma_{21}}}, \frac{\lambda_{1}^{1-\gamma_{12}} \beta_{1}^{\gamma_{12}}}{\lambda_{1}^{1-\gamma_{12}} \beta_{1}^{\gamma_{12}}+\lambda_{2}^{1-\gamma_{21}} \beta_{2}^{\gamma_{21}}}\right),
$$

where the explicit dependencies of asymptotic states on the transport parameters $\lambda_{1}$ and $\lambda_{2}$ are evident. In fact, the ratio of the two uniform densities in the limit $t \rightarrow \infty$ is

$$
\frac{n_{1}^{\infty}}{n_{2}^{\infty}}=\frac{\lambda_{2}^{1-\gamma_{21}} \beta_{2}^{\gamma_{21}}}{\lambda_{1}^{1-\gamma_{12}} \beta_{1}^{\gamma_{12}}}=K
$$

where $K$ becomes dependent on the transport parameters $\lambda_{1}$ and $\lambda_{2}$ [see Eq. (2) for comparison]. Note, however, this new constant $K$ cannot be considered an equilibrium constant since in the non-Markovian case considered here a thermodynamic equilibrium state cannot be defined.

\section{B. Survival-extinction phenomenon}

Let us assume $\beta_{1}<\beta_{2}$; then the MAL (2) predicts $n_{1}^{\infty}$ $>n_{2}^{\infty}$. On the contrary, it is clear from Eq. (10) that one could choose the rates of jumping $\lambda_{1}$ and $\lambda_{2}$ so that the inequality can be inverted to $n_{1}^{\infty}<n_{2}^{\infty}$. We obtain even more dramatic results if we take the limit $\lambda_{1} \rightarrow 0$ or $\lambda_{2} \rightarrow 0$. If, for example, we consider the limit $\lambda_{1} \rightarrow 0\left(\lambda_{2} \neq 0\right)$, then one can observe the extinction of particles in state $B$ and survival of particles in state $A$, that is, $n_{1} \rightarrow 1$ and $n_{2} \rightarrow 0$ as $t \rightarrow \infty$ [see (9)]. So we find from our model that transport process can induce a survival and/or extinction of one of the two densities for anomalous reactions. To validate this phenomenon we have performed the direct Monte Carlo simulations of two-states random walks. We model the evolution of individual particles as follows: At $t=0$, every particle is assigned two random waiting times, one for switching to the other state and one for jumping to a new position. These times are distributed according to the pdf's for switching and jumping, respectively. According to our random walk model, only the minimum of two random times is chosen. So, the particle either switches or jumps depending on what the minimum waiting time is, and after that the process starts again. To calculate the average density, around $10^{5}$ particles have been used in our simulations. Since we are interested only in the temporal dynamics of the average density of the particles, the spatial component is not explicitly considered in the 


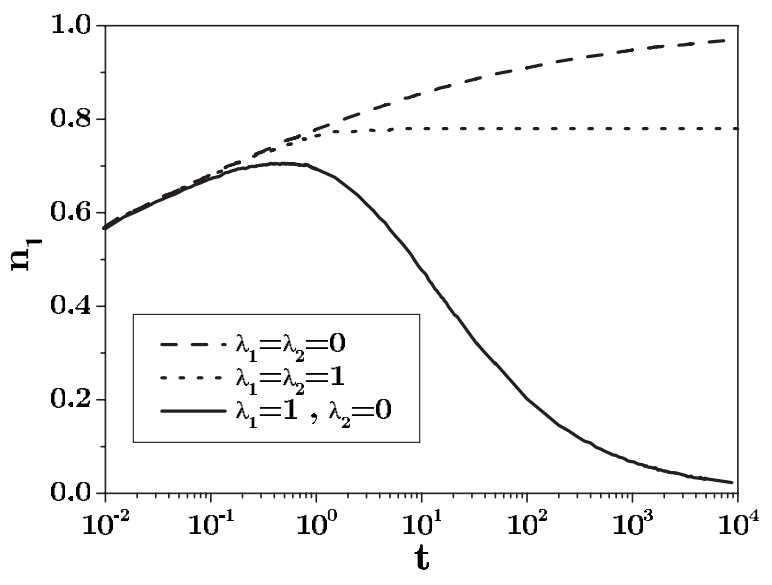

FIG. 1. Time evolution of the density $n_{1}$ obtained from Monte Carlo simulations for different values of the parameters $\lambda_{1}$ and $\lambda_{2}$; $\sigma_{1}^{2}=\sigma_{2}^{2}=1, \beta_{1}=\beta_{2}=1, \gamma_{12}=0.25, \gamma_{21}=0.5$.

simulations. The results are illustrated in Fig. 1 where one can see that if $\lambda_{1} \neq 0$ and $\lambda_{2}=0$, then we might observe the temporal growth of $n_{1}$ before the final decay to zero (solid line). However, if we set $\lambda_{1}=\lambda_{2}=0$ (dashed line), then the limit for the density of particles in state $A$ is completely different, that is, $n_{1} \rightarrow 1$ as $t \rightarrow \infty$.

The result $n_{i} \rightarrow 1$ as $\lambda_{i} \rightarrow 0$ imply that if the particles do not move in one of the states, they survive. This idea of "staying quiet helps you to surviv" can be understood from the interplay between the waiting times for reactions and jumps. According to our derivation, the reaction process, say the transition from state $A$ to state $B$, is actually governed by the density $\Phi_{1} \psi_{12}$ [the particles react only if they have not jumped before, as can be seen from Eq. (4)]. We can refer to this function as the effective waiting time pdf. For an anomalous switching process with Markovian transport the asymptotic behavior of the effective pdf reads as $\Phi_{1} \psi_{12}$ $\sim t^{-1-\gamma_{12}} e^{-\lambda_{1} t}$. Then, the mean waiting time is finite, and for this reason the system reaches a stationary state, given by Eq. (9). However, in the limit $\lambda_{1} \rightarrow 0$ the effective mean waiting time diverges, which makes the reaction $A \rightarrow B$ much slower than the backward reaction $B \rightarrow A$, so the particles tend to get trapped in the state $A$. For this reason we obtain $n_{1} \rightarrow 1$ and $n_{2} \rightarrow 0$ in the long-time limit.

For anomalous transport and anomalous reaction (switching), we have $\psi_{i j}(s) \sim 1-\left(\beta_{i}^{-1} s\right)^{\gamma_{i j}}$ and $\varphi_{i}(s) \sim 1-\left(\lambda_{i}^{-1} s\right)^{\gamma_{i}}$, with $\gamma_{i}<1$ and $\gamma_{i j}<1$. It is helpful again to use the idea of an effective waiting time distribution for reaction, $\Phi_{i} \psi_{i j}$ $\sim t^{-1-\gamma_{i}-\gamma_{i j}}$. So that, the effective mean waiting time can be finite, provided that the condition $\gamma_{i}+\gamma_{i j}>1$ is satisfied. Figure 2 shows the phase diagram for asymptotic states $n_{1}^{\infty}$ and $n_{2}^{\infty}$ depending on the values of $\gamma_{1}+\gamma_{12}$ and $\gamma_{2}+\gamma_{21}$. If both of them are larger than 1 , then the transitions $1 \rightarrow 2$ and $2 \rightarrow 1$ are governed by finite mean waiting times, so a coexistence of two states is possible. In other regions, the divergences of the mean waiting times make the particles get trapped in the state where the switching process is slower. Therefore, one

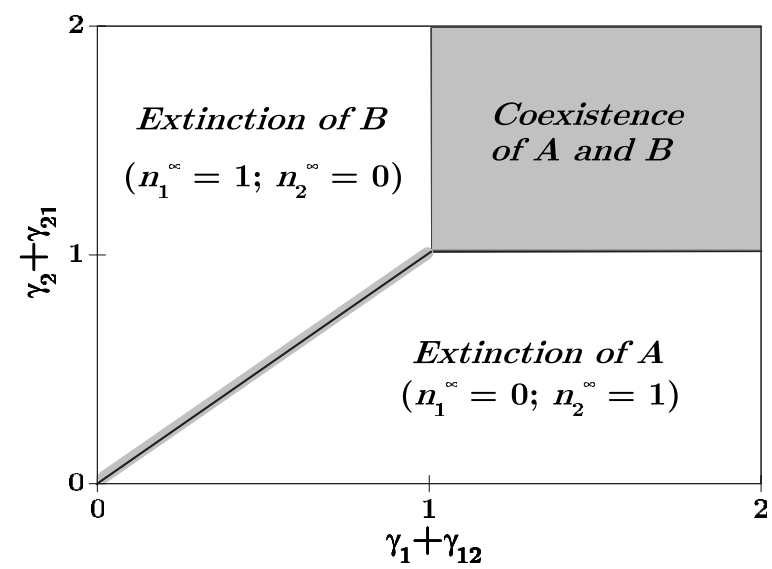

FIG. 2. Phase diagram of the extinction-survival regions for anomalous switching and transport in terms of the exponents $\gamma_{i}$ and $\gamma_{i j}$

of the states becomes extinct in the asymptotic regime. These results can be explained by a coupled renewal property assumed in our model. If the internal waiting time of the particles starts from zero after each event (reaction or jump), then we have a competition between both processes to be the first to occur, and so coupled effects emerge. This coupled renewal property is opposite to additive renewal property where the random walk in space is completely independent of the reaction process (see, for example, [7]).

\section{CONCLUSIONS}

We have presented a non-Markov model for the reversible reaction $A \rightleftarrows B$ and studied the interplay between the anomalous transport and anomalous reaction processes implemented in a probabilistic way. Thus, we have been able to explore those situations that are beyond mass action law. We have derived mesoscopic integro-differential equations for the mean densities of particles in states $A$ and $B$ when the transitions between two states $A$ and $B$ and jumps in space are governed by heavy-tailed waiting-time distributions. It has been shown that the anomalous properties of the reversible reaction yield the appearance of unusual properties such as dependence of asymptotic states on transport and the transient growth of densities before decay. We have found that the transport process can modify completely the uniform stationary regimes. In particular, it can induce the survivalextinction of one of the states. These results have been validated by direct Monte Carlo simulations of a two-state random walk.

\section{ACKNOWLEDGMENTS}

This research has been partially supported by the Generalitat de Catalunya Grant No. 2006-BP-A-10060 (D.C.), and by Grants No. FIS 2006-12296-C02-01, No. SGR 200500087 (V.M.), and No. EPSRC EP/D03115X/1 (S.F., V.M.). 
[1] J. Keizer, Thermodynamics of Nonequilibrium Processes (Springer-Verlag, New York, 1987).

[2] J. D. Murray, Mathematical Biology, 3rd ed. (Springer-Verlag, New York, 2002).

[3] R. Metzler and J. Klafter, Phys. Rep. 339, 1 (2000).

[4] S. B. Yuste and K. Lindenberg, Phys. Rev. Lett. 87, 118301 (2001); Chem. Phys. 284, 169 (2002).

[5] K. Seki, M. Wojcik, and M. Tachiya, J. Chem. Phys. 119, 2165 (2003); 119, 7525 (2003).

[6] S. Fedotov and A. Iomin, Phys. Rev. Lett. 98, 118101 (2007).

[7] I. M. Sokolov, M. G. W. Schmidt, and F. Sagués, Phys. Rev. E 73, 031102 (2006); D. Froemberg and I. M. Sokolov, Phys. Rev. Lett. 100, 108304 (2008); F. Sagués, V. P. Shkilev, and I. M. Sokolov, Phys. Rev. E 77, 032102 (2008).

[8] S. Fedotov and V. Méndez, Phys. Rev. E 66, 030102(R) (2002); A. Yadav, S. Fedotov, V. Méndez, and W. Horsthemke, Phys. Lett. A 371, 374 (2007).

[9] A. Yadav and W. Horsthemke, Phys. Rev. E 74, 066118 (2006).
[10] B. I. Henry, T. A. M. Langlands, and S. L. Wearne, Phys. Rev. E 74, 031116 (2006).

[11] T. A. M. Langlands, B. I. Henry, and S. L. Wearne, Phys. Rev. E 77, 021111 (2008).

[12] I. Goychuk and P. Hänggi, Phys. Rev. E 70, 051915 (2004).

[13] R. Voituriez, M. Moreau, and G. Oshanin, Europhys. Lett. 69, 177 (2005); A. B. Doktorov and A. A. Kipriyanov, J. Phys.: Condens. Matter 19, 065136 (2007).

[14] I. Goychuk and P. Hänggi, Phys. Rev. Lett. 91, 070601 (2003).

[15] X. Brokmann, J. P. Hermier, G. Messin, P. Desbiolles, J. P Bouchaud, and M. Dahan, Phys. Rev. Lett. 90, 120601 (2003).

[16] E. Barkai and Y.-C. Cheng, J. Chem. Phys. 118, 6167 (2003); F. Barbi, M. Bologna, and P. Grigolini, Phys. Rev. Lett. 95, 220601 (2005); I. M. Sokolov and J. Klafter, ibid. 97, 140602 (2006); O. Ehlert et al., Mikrochim. Acta 160, 351 (2008).

[17] A. I. Shushin, Phys. Rev. E 64, 051108 (2001).

[18] A. Giese et al., Int. J. Cancer 67, 275 (1996); A. Giese et al., J. Clin. Oncol. 21, 1624 (2003).

[19] J. R. Cann, Anal. Biochem. 237, 1 (1996). 